\title{
Respiratory Care in Children with COVID-19
}

\author{
Shalu Gupta ${ }^{1}$ Suresh K. Angurana ${ }^{2}$ Virendra Kumar ${ }^{3}$ \\ ${ }^{1}$ Department of Pediatric, Lady Hardinge Medical College and \\ Kalawati Saran Children's Hospital, New Delhi, India \\ 2 Department of Pediatrics, Advanced Pediatrics Centre (APC), \\ Postgraduate Institute of Medical Education and Research, \\ Chandigarh, India \\ ${ }^{3}$ Department of Pediatric, Lady Hardinge Medical College and \\ Kalawati Saran Children's Hospital, New Delhi, India \\ J Pediatr Intensive Care 2023;12:87-93.
}

\begin{abstract}
Keywords

- COVID-19

- noninvasive ventilation

- high-flow nasal cannula

- personal protective equipment

- mechanical ventilation
\end{abstract}

\begin{abstract}
Address for correspondence Shalu Gupta, MD, DM, Division of Pediatric Emergency and Critical Care, Department of Pediatrics, Lady Hardinge Medical College and Kalawati Saran Children's Hospital, New Delhi 110001, India (e-mail: sguptabhu@gmail.com).
\end{abstract}

The novel coronavirus disease 2019 (COVID-19) pandemic caused by severe acute respiratory syndrome coronavirus- 2 (SARS-CoV-2) has overwhelmed the health care systems and crippled economies around the world. Since December 2019, countries after countries have become victim of the COVID-19 pandemic. According to the initial studies, nearly 3 to $10 \%$ of the patients required intensive care unit (ICU) admission. ${ }^{1}$ In adult patients, the most common causes of ICU admission were

received

October 15, 2020

accepted after revision

December 20, 2020

article published online

February 11, 2021 acute hypoxemic respiratory failure with or without severe hypercapnia from acute respiratory distress syndrome (ARDS; $60-70 \%)$, followed by shock (30\%), myocardial dysfunction (20-30\%), and acute kidney injury (AKI; $10-30 \%)^{2}$ In a review of 55,924 confirmed adult cases from China by the World Health Organization (WHO)-China Joint Mission, 13.8\% were classified as severe and $6.1 \%$ as critical (respiratory failure, shock, and multiple organ dysfunction syndrome [MODS]). ${ }^{3}$

Since there is no definitive treatment for COVID-19, the only treatment is supportive management of patients with

(c) 2021. Thieme. All rights reserved. Georg Thieme Verlag KG, Rüdigerstraße 14,

DOI https://doi.org/ 10.1055/s-0041-1723036. 70469 Stuttgart, Germany
ISSN $2146-4618$. 
COVID-19. ${ }^{1}$ Since lungs are the predominant organs involved in COVID-19, and respiratory support is the most common intervention, it should be provided taking all precautions to limit transmission of virus to health care providers (HCPs) and ensuring safety of the patients.

\section{Pediatric COVID-19}

Pediatric COVID-19 is relatively mild disease when compared with adults and accounts for 1 to $5 \%$ of the case load. ${ }^{4}$ Data suggest that Pediatric COVID-19 cases might be less severe than adults and that children might experience different symptoms than adults. ${ }^{4-8}$ According to previous studies, the involvement of children is as follows: asymptomatic (4-21\%), mild (51-58\%), moderate (19-39\%), severe (1-5.2\%), and critical disease (0.6-1.7\%). The children with oxygen saturation $<92 \%$ were in range of 1 to $2.3 \%$ and very few required assisted-mechanical ventilation. The mortality rate in children ranged from 0 to $0.6 \%{ }^{5,6,8,9}$

In a retrospective Chinese study on the epidemiological characteristics of 2143 Pediatric patients with COVID-19, it was demonstrated that $34.1 \%(n=731)$ cases were laboratory-confirmed and young children, particularly infants, were vulnerable to SARS CoV-2 infection. The proportion of severe and critical cases was $10.6,7.3,4.2,4.1$, and $3.0 \%$ for the age group of $<1,1$ to 5,6 to 10,11 to 15 , and 16 years, respectively. Only one 14 -year-old boy died. ${ }^{6}$ Among 149,082 cases of COVID-19 reported from the United States, $2,572(1.7 \%)$ were aged $<18$ years. ${ }^{10}$ Hospitalization status was available for 745 (29\%) cases in children aged $<18$ years and 35,061 (31\%) in adults aged 18 to 64 years. Among children with COVID-19, 147 (5.7-20\%) were reported to be hospitalized and $15(0.58-2 \%)$ were admitted to an ICU. Children aged $<1$ year accounted for the highest percentage (15-62\%) of hospitalization among pediatric patients with COVID-19. There were three deaths in this report. ${ }^{10}$ Similarly, in Madrid, Spain, during the first 2 weeks of the epidemic, $60 \%$ (25/41) children with confirmed COVID-19 were hospitalized, 9.7\% (4/41) were admitted to ICU, and 9.7\% (4/41) needed respiratory support beyond nasal prongs. Only one kid required mechanical ventilation and no death was reported. $^{11}$

This fact is further reiterated in one of the recently published systematic review of Pediatric cases of COVID19 , which demonstrated that most children and adolescents presented with mild symptoms. ${ }^{12}$ Out of 1,065 children with SARS-CoV-2 infection, respiratory symptoms were mild except in one 13-month-old infant who presented with severe lower respiratory tract infection (COVID-19 pneumonia), complicated by shock and kidney failure, required ICU and invasive ventilation. ${ }^{13}$ No Pediatric death from COVID19 was reported in the age range of 0 to 9 years. As compared with adults, children with COVID-19 showed a better prognosis and recovered within 1 to 2 weeks. ${ }^{12}$ Children with chronic diseases, like bronchopulmonary dysplasia and cystic fibrosis, were also less infected than adults. ${ }^{14}$

In late April 2020, clinicians from the United Kingdom (UK) reported a cluster of eight previously healthy children who presented with hyperinflammatory shock syndrome temporally associated with COVID-19. ${ }^{15}$ Thereafter, multiple reports of pediatric inflammatory multisystem syndrome temporally associated with SARS-CoV-2 (PIMS-TS), multisystem inflammatory syndrome in children (MIS-C), Kawasaki's disease (KD), and Kawasaki's-like syndrome were published from the countries with high case load of COVID-19 like the UK, France, Italy, and the United States. ${ }^{16-19}$

The peak in the cases with PIMS-TS or MIS-C followed 2 to 6 weeks after the peak of COVID-19 pandemic. ${ }^{16-19}$ The age group involved was of older children and the most common presenting features were fever, gastrointestinal symptoms, rash, conjunctival injection, and respiratory symptoms. Majority had positive SARS-CoV-2 antibody test and one-third had positive reverse-transcription polymerase chain reaction (RT-PCR). The commonest laboratory abnormalities were elevated C-reactive protein (CRP), D-dimer, procalcitonin, brain natriuretic peptide (BNP), fibrinogen, ferritin, troponin, and interleukin-6 (IL-6); lymphopenia, hypoalbuminemia, and thrombocytopenia. The cardiovascular manifestations noted were shock (60-65\%), myocardial dysfunction (30-60\%), myocarditis (65\%), and coronary artery abnormalities (30-40\%). About 65 to $70 \%$ required pediatric intensive care unit (PICU) admission, 40 to $60 \%$ vasoactive drugs, and 15 to $25 \%$ required mechanical ventilation. The common treatment provided was intravenous immunoglobulin (IVIg; 65-80\%), steroids (50-60\%), antiplatelet drugs (64\%), and anticoagulation (50-55\%). The overall mortality was low (1-2\%). ${ }^{18,20-25}$

\section{Radiological Assessment in Children}

The chest computed tomography (CT) manifestations of COVID-19 in pediatric cases are diverse and lack specificity. As compared with adults, ground glass opacities (GGOs) in pediatric cases with COVID-19 are more localized with lower attenuation, and less lobular involvement. Other CT manifestations noted are consolidation, GGOs with consolidation, or interlobular septa thickening. ${ }^{26}$ However, the radiological picture often gets complicated by the underlying coinfection in children. ${ }^{27}$ Moreover, there are some pediatric cases with positive RT-PCR presenting with normal CT scans. Therefore, CT provides an unnecessary risk of exposure to radiation, therefore must be chosen with extreme caution in children. It is also unclear whether CT scanning has any additional value as a screening tool to rule out COVID-19 infections in children with little or no respiratory symptoms or with negative or missing RT-PCR test results. ${ }^{28}$ Even in adult population, the sensitivity of a chest CT to detect abnormalities in proven COVID-19 patients ranges from 44 to $97 \%$ (median, 69\%). ${ }^{28}$

The evidence for point of care lung ultrasound (POCUS) in COVID-19 has grown in this pandemic, although it mainly includes case reports, opinion pieces, and tutorials. ${ }^{29}$ POCUS has a high sensitivity for the pulmonary manifestations of COVID-19, such as ARDS and consolidation. ${ }^{29}$ POCUS could also be utilized to monitor treatment response. Furthermore it's an easy available bed-side tool and minimizes nosocomial 
spread of the disease. ${ }^{29,30}$ In a meta-analysis of seven studies (122 patients), almost all patients had abnormal lung ultrasound. ${ }^{31}$ The common abnormalities were interstitial involvement/B-pattern (97\%), pleural line abnormalities (70\%), pleural thickening (54\%), consolidation (39\%), and pleural effusion (14\%). ${ }^{31}$ Thus there is a potential role of this modality in the triage, diagnosis, and follow-up of COVID-19 patients.

\section{Why COVID-19 is Less Frequent and Less Severe in Children?}

The answer to this relevant question is not clear and several hypotheses have been postulated related to both exposure and host factors. Children are having less exposure of COVID19 cases, as they are usually well cared for at home, the schools are closed, and outdoor activity is limited. ${ }^{6}$ Children may be less susceptible to SARS-CoV-2 as a result of possible age-related differences in the expression of angiotensinconverting enzyme 2 (ACE2) receptors. ${ }^{32}$ Adults who required ICU care had higher levels of cytokines, whereas children with COVID-19 showed a lower degree of immune dysregulation in contrast to adults. Lymphopenia is a common finding in adults, whereas it was noted in only 3.5\% of pediatric cases in the Wuhan Children's Hospital Series. ${ }^{5}$ Furthermore, as the children's immune system is evolving, it might respond to different viral antigens differently. Also, frequent intercurrent viral infections and vaccination could possibly enhance the immunity and play a protective role against developing COVID-19 in children.

\section{Transmission from Children to Adults and Caregivers}

The current literature is limited regarding risk of SARS-CoV-2 transmission from children to other children, adults, and caregivers. Children usually have mild or asymptomatic disease; transmission has been demonstrated to occur from asymptomatic, as well as symptomatic individuals, including transmission from children to adults within family clusters. ${ }^{33,34}$ Although SARS-CoV-2 is thought to be predominantly transmitted by respiratory droplets, fecal-oral transmission is also possible. ${ }^{35}$ It is unclear whether fecal RT-PCR represents active viral replication or residual noninfectious viral genomic material and the potential risk for transmission. ${ }^{34,35}$ However, there are contradictory reports which suggested that children have not played a significant role in household transmission. ${ }^{36,37}$ The data on school-related transmission is also very limited. In a systematic review based on 69 pediatric cases, the mean duration of viral shedding through the respiratory tract was $11.1 \pm 5.8$ days and from gastrointestinal tract was $23.6 \pm 8.8$ days from symptom onset. In $89 \%$ of cases, viral shedding via the gastrointestinal tract persisted for 4 weeks after nasopharyngeal or throat swabs became negative. ${ }^{35}$ Further studies are required to determine whether children are less or more infectious than adults. Till then, standard prevention measures should be diligently followed.

\section{Personal Protective Equipments}

For HCPs performing aerosol-generating procedures (AGPs; taking respiratory samples, nebulization, endotracheal intubation, bag and mask ventilation, endotracheal suctioning, noninvasive ventilation [NIV], tracheostomy, cardiopulmonary resuscitation, laryngoscopy, and bronchoscopy) on COVID-19 patients, it is recommended to use well-fitted respirator masks (N95 respirators, filtering face piece 2 , or equivalent) in addition to other PPE (gloves, fluid-resistant full body gown or apron, eye protection, such as a face shield or safety goggles, and shoe covers). ${ }^{38-40}$ AGPs should be performed in single-patient negative pressure room or single-patient well-ventilated room. It is important to train HCPs in donning and doffing of PPE.

\section{Respiratory Support in Children with COVID-19}

As evident from the literature, children are less commonly affected by COVID-19, the severity of disease is less, and mortality is $<1 \%$. Therefore, the data on requirement of respiratory support and mechanical ventilation is scarce in the pediatric population. There are currently no randomized clinical trial to guide treatment of these very few children who presented with life-threatening complications, such as severe pneumonia, ARDS, sepsis, and septic shock, in context of COVID-19. Most of the data and guidelines are extrapolated from the literature and guidelines on adults with COVID19 , evidence from other viral respiratory infections, and general intensive care management guidelines. ${ }^{1,39}$

\section{Supplemental Oxygen}

In adults with COVID-19, the guidelines suggest starting supplemental oxygen if the peripheral oxygen saturation (SpO2) is $<92 \%$ on room air, and recommend starting supplemental oxygen if $\mathrm{SpO} 2$ is $<90 \%$. It is recommended that in adult patients with acute hypoxemic respiratory failure on oxygen, the saturation should be maintained no higher than $96 \%{ }^{39}$

In children with COVID-19 with respiratory distress and/or $\mathrm{SpO}_{2}<90 \%$, oxygen can be started with low flow devices according to tolerability by the patient, familiarity by the treating team, availability, amount of oxygen needed $\left(\mathrm{FiO}_{2}\right)$, and cost. ${ }^{40}$ The nasal prongs (1-5 L/min), nasal cannula, face-mask, venturi mask, nonrebreathing mask, or bubble continuous positive airway pressure (bCPAP) can be used based on above-mentioned parameters. High-flow oxygen delivery devices have potential for aerosol generation. If tolerated, children can be advised to wear triple layer surgical mask over the nasal prongs or nasal cannula. ${ }^{41}$

\section{Noninvasive Ventilation and Heated Humidified High-Flow Nasal Cannula}

In critically ill adult patients with COVID-19 in China, the use of NIV and HFNC was about one-third and two-thirds, 
respectively. ${ }^{1}$ The main concern in using these devices was the increased risk of exposure to HCPs due to aerosol generation, if negative pressure rooms are not available. The literature is ambiguous regarding the safety concerns as regards the aerosol generation. Though previous data suggest that NIV was associated with nosocomial transmission in cases of SARS, ${ }^{42}$ on the contrary human laboratory data suggest that NIV does not generate aerosols. ${ }^{43}$

There are several pediatric masks available with multiple sizes, facilitating NIV therapy in children. Commonly used mask interface for NIV in children includes nasal mask, oronasal mask, nasal pillows, and total-faced masks. ${ }^{41} \mathrm{Mul}$ tiple factors require consideration in the selection of an interface including age and developmental stage, facial anatomy, a child's personal mask choice, interface availability, and available NIV machine. Careful mask selection, a wellfitting headgear, and time investment for mask desensitization are some important recommendations for adequate mask adaptation in children. ${ }^{44}$ Data in children comparing performance among various mask interfaces is scarce. In one retrospective study involving 62 children ( $>2$ years of age), no difference was seen in NIV adherence, correction of abnormalities in nocturnal gas exchange, and leak values between nasal pillows, nasal, and oronasal masks. ${ }^{45}$

Similarly, there might be safety issues with the use of HFNC. Studies that evaluated the safety of HFNC were not designed to show whether or not HFNC is AGP and did not examined the spread of viruses. ${ }^{46}$ HFNC does not seem to confer an increased risk of transmission of disease. In studies, evaluating bacterial environmental contamination, HFNC presented as a contamination risk similar to that of conventional oxygen. ${ }^{47}$ If we take evidence from studies during the SARS epidemic, HCPs exposed to HFNC were not at increased risk of developing disease. ${ }^{48}$ Also, HFNC was more comfortable than the conventional oxygen therapy. ${ }^{49}$ Therefore, in adults with COVID-19 and acute hypoxemic respiratory failure, guidelines prefer HFNC over conventional oxygen therapy. ${ }^{40}$

However, in children with COVID-19 who persist to have increased work of breathing and hypoxemia on supplemental oxygen should receive HFNC if available. Patients with progressive respiratory distress, or where HFNC is unavailable, can be escalated to NIV, bCPAP, or bilevel positive airway pressure (BiPAP)..$^{40}$

A systematic review which included 12 randomized controlled trials (RCTs; 1,989 patients), provided low-certainty evidence that HFNC may reduce invasive ventilation (relative risk $[R R]=0.85 ; 95 \%$ confidence interval $[\mathrm{CI}]$ : 0.74-0.99) and escalation of oxygen therapy $(\mathrm{RR}=0.71 ; 95 \% \mathrm{CI}$ : $0.51-0.98)$ in patients with respiratory failure ${ }^{50}$ No difference in mortality was seen between patients receiving HFNC versus conventional oxygen therapy. ${ }^{50}$

Although NIV might reduce intubation and mortality in mild ARDS, it is associated with higher mortality in moderate-to-severe ARDS from multiple causes. In one trial, using NIV, failure was reported in $49 \%$ of patients with hypoxic respiratory failure requiring escalation to intubation. ${ }^{51}$ In addition, patients with hypoxic respiratory failure random- ized to NIV had higher mortality (28\%; 95\% CI: $21-37 \%$ ) as compared with the conventional oxygen therapy (23\%; $95 \%$ CI: $16-33 \%)$ or $\mathrm{HFNC}(13 \%$; $95 \% \mathrm{CI}: 7-20 \% ; p=0.02) .{ }^{51}$ In another cohort of Middle East Respiratory Syndrome (MERS) adult patients, NIV was associated with a high failure rate (92.4\%) leading to intubation. ${ }^{52}$

Moreover, NIV may generate large tidal volumes and greater transpulmonary pressures, further aggravating the already injured lung. Also, the delayed initiation of invasive mechanical ventilation may lead to emergency or more unstable intubation that increases the risk of aerosol transmission. ${ }^{53}$

In children, HFNC or NIV are safe and efficacious modes of respiratory support may provide adequate respiratory support to prevent the need for invasive mechanical ventilation. ${ }^{40}$

However, patient on noninvasive mode of ventilation (HFNC or NIV) should be monitored closely for a possible deterioration. In case of nonimprovement or deterioration, early intubation should be planned in a controlled setting rather than waiting for long time and then performing highrisk intubation in emergency and uncontrolled setting which may increase the risk of nosocomial infection to HCPs. ${ }^{52}$

Thus, HFNC and NIV may be reserved for children with mild ARDS without hemodynamic instability, with strict close monitoring, airborne precautions, and in single patient rooms with negative pressure. To limit environmental contamination, the nonvented masks as interface, double lumen tubings, and viral filter at expiratory limb should be used while delivering NIV. There should be low thresholds for intubation and invasive mechanical ventilation in the event of deterioration while on HFNC or NIV.

\section{Endotracheal Intubation}

Children with worsening clinical status, respiratory fatigue, hemodynamic instability, $\mathrm{PaO}_{2} / \mathrm{FiO}_{2}<300$, or altered mental status should be considered for early intubation and mechanical ventilation. ${ }^{41}$ Since intubation is an AGP, previous studies on SARS and MERS showed that intubation poses a risk of viral transmission to HCPs. ${ }^{1,54}$ Therefore, it is of utmost importance to maintain safety of patient, as well as of HCPs, while performing endotracheal intubation.

The endotracheal intubation should be performed in negative pressure room or in single-patient well-ventilated room. Use full PPE along with N95 or equivalent respirators and perform hand hygiene. ${ }^{38,39}$ The checklist for drugs and equipments, and clear roles should be in place, and ventilator should be ready and on stand-by mode with disposable tubing with viral filter between expiratory limb of the circuit and machine. Inside the room, limit the number of staff to three-four (intubator, airway assistant, nurse for administering medication, and team leader). The most skilled or experienced operator should perform the endotracheal intubation to minimize the number of intubations attempts and risk of transmission. ${ }^{38}$ The necessary plan should be communicated and alternative plan for the difficult airways should be ready. It is preferable to perform endotracheal intubation using videoguided laryngoscopy to reduce the distance between patient's 
airway and the HCP. ${ }^{8,38}$ The transparent aerosol entrainment box or plastic sheets can be used to cover the patients head and upper body while intubation to limit exposure to aerosols. These can also be used during extubation.

Since bag and mask ventilation generates aerosols, the preoxygenation can be done with nonrebreathing mask or tight-fitted face mask and bag with high-efficiency particulate air (HEPA) filter between face mask and bag but without providing positive pressure breaths. ${ }^{38}$ If positive pressure breaths are needed, create a tight mask seal (two-hand technique by one HCP and bagging by other) and provide positive pressure breaths at minimal rate and with small tidal volume. ${ }^{54}$ The cuffed or microcuffed endotracheal tubes should be used to limit peritubal air leak and risk of transmission. Use of rapid sequence induction with muscle relaxants will reduce coughing and prevent transmission to the HCPs. Once intubated, immediately inflate the cuff, connect the endotracheal tube to the already set ventilator with closed in-line suction and viral filter between endotracheal tube and circuit, and turn on the ventilator. Observation of chest rise, end-tidal carbon dioxide detection, or lung ultrasound should be used to confirm endotracheal tube placement. Use in-line closed suctioning systems postintubation to reduce aerosol generation. The unnecessary disconnections from the ventilator should be avoided. In case of circuit disconnection, the tube should be immediately clamped and connection should be reestablished. After intubation, all surfaces should be cleaned with $1 \%$ sodium hypochlorite, equipment's should be properly cleaned or disposed, and doffing which should be monitored by trained observer or buddy. ${ }^{8}$ Since the process is complex and needs a lot of coordination, it is suggested to run regular simulation session in the unit. ${ }^{8}$

\section{Mechanical Ventilation}

The mechanical ventilation strategies in children with COVID-19 and ARDS are more or less similar to any other child with ARDS due to other cause except for the aerosol precautions and infection control precautions to limit spread of infection to HCPs. The major focus of mechanical ventilation for COVID-19 is the avoidance of ventilator-induced lung injury while facilitating gas exchange via lung-protective ventilation. It is recommended to use low tidal volume (Vt) ventilation (Vt, $4-8 \mathrm{~mL} / \mathrm{kg}$ of predicted body weight), over higher tidal volumes $(\mathrm{Vt}>8 \mathrm{~mL} / \mathrm{kg}){ }^{39,55}$ Target plateau pressures (Pplat) should be kept $<30 \mathrm{~cm}$ of $\mathrm{H}_{2} \mathrm{O}$ when ventilating patients with ARDS. There are no clinical trials examining the effect of positive end-expiratory pressure (PEEP) on coronavirus-induced ARDS. However, for mechanically ventilated adults with COVID-19 with moderate-to-severe ARDS, guidelines suggest using a higher PEEP strategy over a lower PEEP strategy, keeping check on barotrauma. ${ }^{39}$ There is no defined limit of lower or higher PEEP; however, any PEEP levels $>10 \mathrm{~cm}$ of $\mathrm{H}_{2} \mathrm{O}$ constitutes a higher PEEP strategy. Start with PEEP of 7 to $10 \mathrm{~cm}$ of $\mathrm{H}_{2} \mathrm{O}$ and increase to 10 to $15 \mathrm{~cm}$ of $\mathrm{H}_{2} \mathrm{O}$.

Other strategies include adequate sedation and analgesia, neuromuscular blockade, restrictive fluid strategy, prone positioning, permissive hypoxemia to limit $\mathrm{FiO}_{2}<60 \%$, and permissive hypercapnia. ${ }^{41}$ Judicious use of neuromuscular blocking agents (NMBA), either intermittent or continuous infusion till 48 hours should be used to facilitate lung protective ventilation. ${ }^{39,56}$ The optimal fluid strategy in COVID19 is not known; however, the strategy should remain the same as in any case of ARDS and use a conservative fluid strategy over a liberal fluid strategy. ${ }^{39}$

Prone positioning should be applied early for 12 to 16 hours/day when $\mathrm{PaO}_{2} / \mathrm{FiO}_{2}<150$, given its association with reduced mortality in moderate to severe ARDS. ${ }^{39,55,57}$ Every unit should have a protocol for prone positioning. Even cardiopulmonary resuscitation is advocated in prone position to minimize aerosolization. ${ }^{58}$ Clinicians should be aware of its complications such as pressure sores, vascular line and endotracheal tube displacement, facial edema, transient hemodynamic instability, corneal abrasions, brachial plexus injury, and hemodialysis vascular access flow issues. ${ }^{39}$ The surviving sepsis campaign guidelines suggests to use corticosteroids in mechanically ventilated adults with COVID-19 and ARDS (weak recommendation). ${ }^{39}$ This decision needs to be taken on case-to-case basis in pediatric population and is not routinely recommended.

In patients with severe ARDS due to COVID-19 not improving on optimizing ventilation and rescue strategies, a trial of inhaled bronchodilator (nitric oxide), recruitment maneuver, and if available, venovenous extracorporeal membrane oxygenator (VV-ECMO) can be used. There are no clinical trials of ECMO in COVID-19 patients. Since the availability of ECMO is questionable in resource-limited centers, and it requires an experienced center with full infrastructure, ECMO should only be considered in carefully selected patients with COVID-19 and severe ARDS. ${ }^{39}$

\section{Nebulization}

Since nebulization also generates aerosols, it should be avoided. If bronchodilators are required, they can be administered by a metered dose inhaler with spacer. ${ }^{38,41}$

\section{Weaning and Extubation}

In children recovering from the disease, the routine weaning and extubation protocols should be followed. During extubation, the transparent aerosol entrainment box or plastic sheets can be used to cover the patients' head and upper body to limit exposure to HCPs. The patient should be extubated to nasal prongs with continuous monitoring to assess any need for HFNC or NIV. ${ }^{41}$

\section{Conclusion}

Children with COVID-19 often need respiratory support which can be provided by simple measures like nasal prongs, nasal cannula, face mask, bCPAP, venturi mask, and nonrebreathing mask. The use of HFNC and NIV should be limited to children with mild ARDS preferably in negative pressure 
rooms and with adequate PPE. Early intubation and invasive mechanical ventilation in children with moderate-to-severe ARDS, and those with hemodynamic compromise, altered sensorium, rapid deterioration, and multiple organ dysfunction syndrome are needed. While performing intubation and mechanical ventilation, every attempt should be made to limit exposure to aerosols. The lung-protective mechanical ventilation strategies should be used with adequate sedation, analgesia, and neuromuscular blockers.

\section{Authors' Contributions}

S.G. and S.K.A. reviewed the literature and prepared the initial draft. V.K. critically evaluated and modified the manuscript. S.G. will act as guarantor of the paper.

\section{Conflict of Interest}

None declared.

\section{References}

1 Mehta Y, Chaudhry D, Abraham OC, et al. Critical care for COVID-19 affected patients: position statement of the Indian Society of Critical Care Medicine. Indian J Crit Care Med 2020;24(04):222-241

2 Phua J, Weng L, Ling L, et al; Asian Critical Care Clinical Trials Group. Intensive care management of coronavirus disease 2019 (COVID-19): challenges and recommendations. Lancet Respir Med 2020;8(05):506-517

3 WHO-China Joint Mission. Report of the WHO-China Joint Mission on Coronavirus Disease 2019 (COVID-19). March 7, 2020 at: https://www.who.int/docs/default-source/coronaviruse/whochina-joint-mission-on-covid-19-final-report.pdf.Accessed

4 Ludvigsson JF. Systematic review of COVID-19 in children shows milder cases and a better prognosis than adults. Acta Paediatr 2020;109(06):1088-1095

5 Lu X, Zhang L, Du H, et al; Chinese Pediatric Novel Coronavirus Study Team. SARS-CoV-2 infection in children. N Engl J Med 2020; 382(17):1663-1665

6 Dong Y, Mo X, Hu Y, et al. Epidemiology of COVID -19 among children in China. Pediatrics 2020;145(06):e20200702

7 Cao Q Chen YC, Chen CL, Chiu CH. SARS-CoV-2 infection in children: Transmission dynamics and clinical characteristics. J Formos Med Assoc 2020;119(03):670-673

8 Ong JSM, Tosoni A, Kim Y, Kissoon N, Murthy S. Coronavirus disease 2019 in critically ill children: a narrative review of the literature. Pediatr Crit Care Med 2020;21(07):662-666

9 Parri N, Lenge M, Buonsenso DCoronavirus Infection in Pediatric Emergency Departments (CONFIDENCE) Research Group. Children with COVID-19 in pediatric emergency departments in Italy. N Engl J Med 2020;383(02):187-190

10 CDC COVID-19 Response Team. Corona virus disease 2019 in children - United States, February 12-April 2, 2020. MMWR 2020;69(14):422-426

11 Tagarro A, Epalza C, Santos M, et al. Screening and severity of coronavirus disease 2019 (COVID-19) in children in Madrid, Spain. JAMA Pediatr 2021;175(03):316-317

12 Castagnoli R, Votto M, Licari A, et al. Severe acute respiratory syndrome coronavirus 2 (SARS-CoV-2) infection in children and adolescents: a systematic review. JAMA Pediatr 2020;174(09): 882-889

13 Chen F, Liu ZS, Zhang FR, et al. [First case of severe childhood novel coronavirus pneumonia in China] (in Chinese). Zhonghua Er Ke Za Zhi 2020;58(03):179-182

14 Stanton BA, Hampton TH, Ashare A. The pathophysiology of COVID-19 and SARS-CoV-2 infection. Am J Physiol Lung Cell Mol Physiol 2020;319(03):L408-L415
15 Riphagen S, Gomez X, Gonzalez-Martinez C, Wilkinson N, Theocharis P. Hyperinflammatory shock in children during COVID-19 pandemic. Lancet 2020;395(10237):1607-1608

16 Feldstein LR, Rose EB, Horwitz SM, et al; Overcoming COVID-19 Investigators CDC COVID-19 Response Team. Multisystem inflammatory syndrome in U.S. children and adolescents. N Engl J Med 2020;383(04):334-346

17 Belot A, Antona D, Renolleau S, et al. SARS-CoV-2-related paediatric inflammatory multisystem syndrome, an epidemiological study, France, 1 March to 17 May 2020. Euro Surveill 2020;25(22): 2001010

18 Dufort EM, Koumans EH, Chow EJ, et al; New York State and Centers for Disease Control and Prevention Multisystem Inflammatory Syndrome in Children Investigation Team. Multisystem inflammatory syndrome in children in New York State. N Engl J Med 2020;383(04):347-358

19 Davies P, Evans C, Kanthimathinathan HK, et al. Intensive care admissions of children with paediatric inflammatory multisystem syndrome temporally associated with SARS-CoV-2 (PIMS-TS) in the UK: a multicentre observational study. Lancet Child Adolesc Health 2020;4(09):669-677

20 Kaushik A, Gupta S, Sood M, Sharma S, Verma S. A systematic review of multisystem inflammatory syndrome in children associated with SARS-CoV-2 infection. Pediatr Infect Dis J 2020;39 (11):e340-e346

21 Aronoff SC, Hall A, Del Vecchio MT. The natural history of SARSCov-2 related multisystem inflammatory syndrome in children (MIS-C): a systematic review. J Pediatric Infect Dis Soc 2020; 112:1-6

22 Ahmed M, Advani S, Moreira A, et al. Multisystem inflammatory syndrome in children: A systematic review. EClinicalMedicine 2020;26:100527

23 Jain S, Sen S, Lakshmivenkateshiah S, et al. Multisystem inflammatory syndrome in children with COVID-19 in Mumbai, India. Indian Pediatr 2020;57(11):1015-1019

24 Dhanalakshmi K, Venkataraman A, Balasubramanian S, et al. Epidemiological and clinical profile of pediatric inflammatory multisystem syndrome - temporally associated with SARS-CoV-2 (PIMSTS) in Indian children. Indian Pediatr 2020;57(11):1010-1014

25 Williams V, Dash N, Suthar R, et al. Clinico-laboratory profile, intensive care needs, treatment details, and outcome of Pediatric inflammatory multisystem syndrome temporally associated with SARS-CoV-2 (PIMS-TS): A systematic review and Meta-analysis. medRxiv 2020. Doi: 10.1101/2020.10.21.20217034

26 Duan YN, Zhu YQ, Tang LL, Qin J. CT features of novel coronavirus pneumonia (COVID-19) in children. Eur Radiol 2020;30(08): 4427-4433

27 Xia W, Shao J, Guo Y, Peng X, Li Z, Hu D. Clinical and CT features in pediatric patients with COVID-19 infection: different points from adults. Pediatr Pulmonol 2020;55(05):1169-1174

28 Merkus PJFM, Klein WM. The value of chest CT as a COVID-19 screening tool in children. Eur Respir J 2020;55(06):200124

29 Kulkarni S, Down B, Jha S. Point-of-care lung ultrasound in intensive care during the COVID-19 pandemic. Clin Radiol 2020;75(09):710.e1-710.e4

30 Soldati G, Smargiassi A, Inchingolo R, et al. Is there a role for lung ultrasound during the COVID-19 pandemic? J Ultrasound Med 2020;39(07):1459-1462

31 Mohamed MFH, Al-Shokri S, Yousaf Z, et al. Frequency of abnormalities detected by point-of-care lung ultrasound in symptomatic COVID-19 patients: Systematic review and meta-analysis. Am J Trop Med Hyg 2020;103(02):815-821

$32 \mathrm{Gu} \mathrm{H}$, Xie Z, Li T, et al. Angiotensin-converting enzyme 2 inhibits lung injury induced by respiratory syncytial virus. Sci Rep 2016; 6:19840

33 Jiehao C, Jin X, Daojiong L, et al. A case series of children with 2019 novel coronavirus infection: clinical and epidemiological features. Clin Infect Dis 2020;71(06):1547-1551 
34 Widders A, Broom A, Broom J. SARS-CoV-2: the viral shedding vs infectivity dilemma. Infect Dis Health 2020;25(03): 210-215

35 Xu CLH, Raval M, Schnall JA, Kwong JC, Holmes NE. Duration of respiratory and gastrointestinal viral shedding in children with SARS-CoV-2: a systematic review and synthesis of data. Pediatr Infect Dis J 2020;39(09):e249-e256

36 Santos VS, Gurgel RQ, Cuevas LE, Martins-Filho PR. Prolonged fecal shedding of SARS-CoV- 2 in pediatric patients: a quantitative evidence synthesis. J Pediatr Gastroenterol Nutr 2020;71(02): 150-152

37 Posfay-Barbe KM, Wagner N, Gauthey M, et al. COVID-19 in children and the dynamics of infection in families. Pediatrics 2020;146(02):e20201576

38 Edelson DP, Sasson C, Chan PS, et al; American Heart Association ECC Interim COVID Guidance Authors. Interim guidance for basic and advanced life support in adults, children, and neonates with suspected or confirmed COVID-19: from the emergency cardiovascular care committee and get with the guidelines-resuscitation adult and pediatric Task Forces of the American Heart Association. Circulation 2020;141(25): e933-e943

39 Alhazzani W, Møller MH, Arabi YM, et al. Surviving sepsis campaign: guidelines on the management of critically ill adults with coronavirus disease 2019 (COVID-19). Intensive Care Med 2020;46(05):854-887

40 Kache S, Chisti MJ, Gumbo F, et al. COVID-19 PICU guidelines: for high- and limited-resource settings. Pediatr Res 2020;88(05): 705-716

41 Sundaram M, Ravikumar N, Bansal A, et al; Intensive Care Chapter of Indian Academy of Pediatrics. Novel coronavirus 2019 (2019$\mathrm{nCoV}$ ) infection: part II - respiratory support in the pediatric intensive care unit in resource-limited settings. Indian Pediatr 2020;57(04):335-342

42 Tran K, Cimon K, Severn M, Pessoa-Silva CL, Conly J. Aerosol generating procedures and risk of transmission of acute respiratory infections to healthcare workers: a systematic review. PLoS One 2012;7(04):e35797

43 Simonds AK, Hanak A, Chatwin M, et al. Evaluation of droplet dispersion during non-invasive ventilation, oxygen therapy, nebuliser treatment and chest physiotherapy in clinical practice: implications for management of pandemic influenza and other airborne infections. Health Technol Assess 2010;14(46): 131-172

44 Castro-Codesal ML, Olmstead DL, MacLean JE. Mask interfaces for home non-invasive ventilation in infants and children. Paediatr Respir Rev 2019;32:66-72

45 Ramirez A, Khirani S, Aloui S, et al. Continuous positive airway pressure and noninvasive ventilation adherence in children. Sleep Med 2013;14(12):1290-1294
46 Hui DS, Chow BK, Lo T, et al. Exhaled air dispersion during highflow nasal cannula therapy versus CPAP via different masks. Eur Respir J 2019;53(04):1802339

47 Leung CCH, Joynt GM, Gomersall CD, et al. Comparison of highflow nasal cannula versus oxygen face mask for environmental bacterial contamination in critically ill pneumonia patients: a randomized controlled crossover trial. J Hosp Infect 2019;101 (01):84-87

48 Raboud J, Shigayeva A, McGeer A, et al. Risk factors for SARS transmission from patients requiring intubation: a multicentre investigation in Toronto, Canada. PLoS One 2010;5(05):e10717

49 Rochwerg B, Granton D, Wang DX, et al. High flow nasal cannula compared with conventional oxygen therapy for acute hypoxemic respiratory failure: a systematic review and meta-analysis. Intensive Care Med 2019;45(05):563-572

50 Agarwal A, Basmaji J, Muttalib F, et al. High-flow nasal cannula for acute hypoxemic respiratory failure in patients with COVID-19: systematic reviews of effectiveness and its risks of aerosolization, dispersion, and infection transmission. Can J Anaesth 2020;67 (09):1217-1248

51 Frat JP, Thille AW, Mercat A, et al; FLORALI Study Group REVA Network. High-flow oxygen through nasal cannula in acute hypoxemic respiratory failure. N Engl J Med 2015;372(23): 2185-2196

52 Alraddadi BM, Qushmaq I, Al-Hameed FM, et al; Saudi Critical Care Trials Group. Noninvasive ventilation in critically ill patients with the Middle East respiratory syndrome. Influenza Other Respir Viruses 2019;13(04):382-390

53 Brochard L, Lefebvre JC, Cordioli RL, Akoumianaki E, Richard JC. Noninvasive ventilation for patients with hypoxemic acute respiratory failure. Semin Respir Crit Care Med 2014;35(04): 492-500

54 Wax RS, Christian MD. Practical recommendations for critical care and anesthesiology teams caring for novel coronavirus (2019-nCoV) patients. Can J Anaesth 2020;67(05):568-576

55 Rimensberger PC, Cheifetz IMPediatric Acute Lung Injury Consensus Conference Group. Ventilatory support in children with pediatric acute respiratory distress syndrome: proceedings from the Pediatric Acute Lung Injury Consensus Conference. Pediatr Crit Care Med 2015;16(05, Suppl 1):S51-S60

56 Alhazzani W, Alshahrani M, Jaeschke R, et al. Neuromuscular blocking agents in acute respiratory distress syndrome: a systematic review and meta-analysis of randomized controlled trials. Crit Care 2013;17(02):R43

57 Munshi L, Del Sorbo L, Adhikari NKJ, et al. Prone position for acute respiratory distress syndrome. a systematic review and metaanalysis. Ann Am Thorac Soc 2017;14(Suppl 4):S280-S288

58 Tiwari L, Taneja LN, Gupta Sand Other Expert Committee Members. IAP ALS update on resuscitation guidelines during COVID-19 pandemic. Indian J Pediatr 2020;27:1-7 